\title{
Review of Service Restoration Methods in Distribution Networks
}

Shen, Feifan; Wu, Qiuwei; Huang, Shaojun; López, Juan Camilo ; Li, Canbing; Zhou, Bin

Published in:

Proceedings of 8th IEEE PES Innovative Smart Grid Technologies Conference

Publication date:

2018

Document Version

Peer reviewed version

Link back to DTU Orbit

Citation (APA):

Shen, F., Wu, Q., Huang, S., López, J. C., Li, C., \& Zhou, B. (2018). Review of Service Restoration Methods in Distribution Networks. In Proceedings of 8th IEEE PES Innovative Smart Grid Technologies Conference IEEE.

\section{General rights}

Copyright and moral rights for the publications made accessible in the public portal are retained by the authors and/or other copyright owners and it is a condition of accessing publications that users recognise and abide by the legal requirements associated with these rights.

- Users may download and print one copy of any publication from the public portal for the purpose of private study or research.

- You may not further distribute the material or use it for any profit-making activity or commercial gain

- You may freely distribute the URL identifying the publication in the public portal

If you believe that this document breaches copyright please contact us providing details, and we will remove access to the work immediately and investigate your claim 


\section{Review of Service Restoration Methods in Distribution Networks}

\author{
Feifan Shen, Qiuwei Wu \\ Department of Electrical \\ Engineering \\ Technical University of \\ Denmark \\ Kgs. Lyngby, 2800 Denmark \\ fshen@elektro.dtu.dk, \\ qw@elektro.dtu.dk
}

\author{
Shaojun Huang \\ Center for Energy Informatics \\ University of Southern \\ Denmark \\ 5230 Odense M, Denmark \\ shahu@mmmi.sdu.dk
}

\author{
Juan Camilo López \\ Department of Energy System \\ University of Campinas \\ Campinas 13083-852, Brazil \\ jclopeza@fee.dsee.unicamp.br
}

\author{
Canbing Li, Bin Zhou \\ College of Electrical and \\ Information Engineering \\ Hunan University \\ Changsha 410082, China \\ licanbing@qq.com \\ binzhou@hnu.edu.cn
}

\begin{abstract}
Service restoration (SR) is one of the most important strategies to improve the resilience of the modern distribution network. After the fault location is identified and isolated, a proper SR plan should be determined to resupply out-of-service areas. This paper reviews the existing methods for SR in distribution networks, which can be categorized into two types, namely centralized methods and decentralized methods, according to their implementation. These methods utilize one or more techniques to derive the SR plan. The used techniques consist of expert systems, heuristic algorithms, meta-heuristic algorithms, graph theory, multi-agent systems and mathematical programming.
\end{abstract}

Index Terms-- Centralized methods, decentralized methods, distribution networks, service restoration.

\section{INTRODUCTION}

Distribution networks supply electric power to customers. Consequently, a failure of any important component of the network will cause an interruption of power supply and discomfort to customers [1]. Therefore, it is of great interest of distribution network operators (DNOs) to enhance the resilience of the network.

The development of the distribution network automation provides new opportunities for the distribution network to realize self-healing. Self-healing is the ability of a network to automatically and intelligently restore itself to the best possible state after a permanent fault occurs [2]. When a fault occurs, the self-healing scheme detects and isolates the fault location, and then uses a service restoration (SR) plan to restore outage areas. After the fault is repaired, the network will return to its original state and the self-healing process ends. SR, as a key aspect of the self-healing process, has received considerable attention from researchers.

The SR problem can be deemed as a system reconfiguration problem, which is implemented by opening some normally closed sectionalizing switches and closing some normally open tie switches. A simple SR plan is illustrated in Fig. 1. After detecting and isolating the fault occurred in zone 1 , the system operator transfers the deenergized loads in zone 2 to the adjacent healthy feeder 2 by closing tie switch 1 (TS1). However, with the increase of size and complexity of the distribution network, many aspects should be considered during the restoration process, such as cold load pick up, minimizing the number of switching operations, overcurrent, etc. [3], which increases the complexity of the SR problem. In addition, the ever-increasing penetration level of distributed energy resources (DERs) brings some new features to SR. For example, distributed generator (DG) units can locally support loads during the restoration process, minimizing the number of switching operations and providing opportunities to restore additional unrestored loads. Moreover, forming microgrids (MGs) can enhance the resilience of the distribution network and allows the network to restore outage areas faster. As shown in Fig. 1, after isolating the fault occurred in zone 5, a MG is embedded and the DG unit is dispatched to support local loads in zone 6.

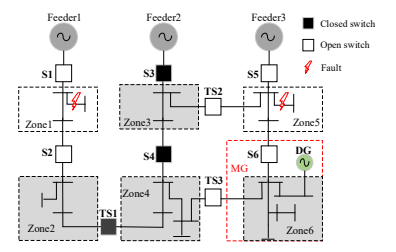

Fig. 1. Service restoration plan of the distribution network

In summary, an effective SR plan should promptly restore as many loads in outage areas as possible while avoiding violating operating constraints.

In this paper, a review of the existing SR methods is presented. According to the implementation, the existing methods can be categorized into two types, namely centralized methods and decentralized methods. These methods use different techniques to derive a feasible or even optimal SR plan. The used techniques include expert systems, heuristic algorithms, meta-heuristic algorithms, graph theory, multiagent systems and mathematical programming.
This work was supported by the DTU-NTU double PhD project of the Smart City joint program. 
This paper is organized as follows. Section II introduces the SR problem. Section III gives an overview of the existing methods for SR. Section IV reviews the existing methods for SR. Section V concludes the paper.

\section{INTRODUCTION FOR SERVICE RESTORATION}

As mentioned before, many aspects of operating objectives and constraints should be considered during the SR process in order to obtain an effective SR plan. According to the reviewed studies, three general objectives are listed as follows:

\section{1) Maximizing priority loads restored}

$$
\max f\left(P_{i}^{l}\right), i \in \Omega_{\text {out }}
$$

where $\Omega_{\text {out }}$ represents the set of all the load buses in outage areas, $P_{i}^{l}$ is the load at $i$ th bus.

\section{2) Minimizing the time required for service restoration$$
\min T_{1}+T_{2}
$$

where $T_{1}$ and $T_{2}$ are the execution time of the restoration algorithm and restoration process, respectively.

\section{3) Minimizing line losses}

$$
\min h\left(I_{i}\right), i \in \Omega_{\text {line }}
$$

where $\Omega_{\text {line }}$ represents the set of all the lines, $I_{i}$ is the magnitude of current flows through ith line.

As the main goal of SR is to resupply de-energized loads, Objective 1) has the top priority. To enhance satisfaction of customers, the time taken for SR should also be minimal, thus the objective 2) should have the second preference. In general, $T_{2}$ can be reduced by minimizing the number of switching operations. For the objective 3), because the target configuration would not last for a long period, loss minimization would not generate a significant benefit. Moreover, this objective may be in conflict with the objectives 1) and 2). Therefore, the objective 3 ) should be the third preference and even disregarded.

To ensure the feasibility of the derived plan, some general operating constraints should be satisfied during the restoration process, such as line current limit constraints, voltage limit constraints, line capacity constraints and radial structure constraint. In addition, when DERs participate in the SR process, some certain operating constraints should be satisfied, such as constraints on the ramp rates of DG units [4], the state of charge levels of batteries of energy storage systems (ESSs) [4] and electrical vehicles (EVs) [5]. It should be noted that Objective 1) is so dominating that some certain constraints, like restrictions on voltages, can be relaxed and even disregarded.

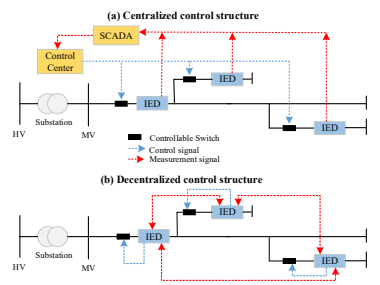

Fig. 2. Structures of centralized and decentralized methods
In summary, the SR problem is a computationally complex problem [6] because it is: 1) combinatorial due to the large number of combinations of possible switching operations; 2) nonlinear due to the nonlinear nature of power flow constraints; 3) multi-objective; 4) constrained due to operating constraints and the radial structure requirement.

\section{AN OVERVIEW OF METHODS FOR SERVICE RESTORATION}

A huge number of methods have been proposed to address the SR problem. According to the implementation, they can be categorized into two types, namely centralized methods and decentralized methods. In centralized methods, all the system operation information is collected by the intelligent electronic devices (IEDs) and transferred to a control center through the communication system. After the fault location is isolated, the control center will implement the restoration algorithm and execute a series of switching actions. However, in decentralized methods, the plan of switching actions is obtained by the communication and coordination between the IEDs that collect local information. The structures of the centralized and decentralized methods are illustrated in Fig. 2.

In the centralized and decentralized frameworks, the SR problem is solved by different techniques, in which expert systems, heuristic algorithms, graph theory and multi-agent systems are used to solve the problem according to the specified rules and procedures derived from the domainspecific knowledge and heuristics, while mathematical programming and meta-heuristic algorithms solve the problem based on the mathematical optimization model.

In addition to the proposed different restoration algorithms, the success of the restoration process also relies on the hardware. The currently-used devices should be upgraded to cope with restoration algorithms. For example, the manually controlled switches (MCSs) should be replaced by the automatic controlled switches (ACSs), and new intelligent devices, such as remote terminal units (RTUs) and intelligent electronic devices (IEDs), are required. Moreover, efficient and reliable two-way data communication links are require to ensure the reliable delivery of communication data.

\section{Methods FOR SERVICE RESTORATION}

In this section, most of the existing methods are presented according to their implementation and techniques used.

\section{A. Centralized Methods}

\section{1) Expert system}

The expert system (ES) is a knowledge based technique, it transfers the expert knowledge into some rules (if-then rule) and uses an inference engine to infer from these rules [7]. The author in [8] developed a knowledge base with 180 rules that are acquired from the literature and discussions with power engineers. The developed ES can implement group restoration, zone restoration and load transfer. A knowledge base was constructed in [9] with rules which divides the outage areas into single group or multiple groups based on feeder margins, branch points and tie switches. An ES was developed in [10] based on a colored petri net inference model that possesses parallel-like inference characteristics. The 
developed ES considers the load priority and efficiently finds a series of proper switching operations. An ES was developed in [11] using the object-oriented programming technique which organizes feeder configuration data into a hierarchy way and improves performance of the inference. Furthermore, the proposed system considers time-dependent loads.

Although the above proposed ESs determine the status of switches efficiently, it is difficult to construct a large knowledge base. Moreover, the maintenance of a large-scale ES is very costly and the optimality of the solution cannot be guaranteed.

\section{2) Heuristic algorithm}

The heuristic algorithm uses domain-specific heuristics to guide the search process. In [12]-[15], different procedures of SR were created based on heuristic rules and translated into programming codes to solve the problem. A heuristic procedure was transformed into a heuristic algorithm in [12], in which the supporting feeders are first used to restore power supply, if the full restoration fails, the loads that can be completely restored by their supporting laterals are restored followed by the loads that cannot be completely restored by their supporting laterals. A multi-tier algorithm was developed in [13]. In the work, tier1 is a set of tie switches, switch pairs and feeders that have links to out-of-service areas, tier 2 is a set of tie switches, switch pairs and feeders that have links to tier1. All switches and feeders in tierl first attempt to restore all de-energized loads, if it fails, tier2 is used to solve the problem. A similar procedure to the one in [13] considering load curtailment was proposed in [14]. The author in [15] developed a load flow based procedure for SR. Firstly, all available switches are treated as ideal current sources and closed to create a meshed network. Then, a secure power flow pattern is conducted to minimize overall system current overloads by adjusting those ideal current sources. Once the magnitude of each current source is known, the switch carrying the least current is opened to eliminate a network loop. The switch is opened in this manner to minimize disturbance in the secure power pattern. After repeating this process, a radial network with acceptable operating requirements can be obtained.

The authors in [16]-[18] constructed the search space as a decision tree and used different search techniques to find the solution. It is noted that the domain-specific knowledge can be used to avoid unnecessary search. The author in [16] developed a binary tree in which each node is a partial assignment of a binary variable (switch). Starting from any parent node, a variable, namely a switch in the network, is selected for branching. Each possible value (0 or 1$)$ of the variable represents a successor node of the parent node. After the binary tree is built, the depth-first search technique is used to search for the desired solution. A different decision tree was proposed in [17], in which a node represents a configuration of the network. The transition from a parent node to a successor node is an available switch pair operation, including opening a sectionalizing switch and closing a tie-switch. In the work, the breadth-first technique is adopted to search the decision tree. The author in [18] built a similar decision tree to [17] without considering load transfers from healthy feeders to other feeders. The $\mathrm{A}^{\text {ast }}$ search technique is used to search a least-cost configuration plan.

In addition, due to the presence of imprecise linguistic terms of heuristic rules, strictly complying with rules may cause some undesired problems, such as unbalanced loads on supporting feeders and infeasible SR issues. Therefore, the fuzzy set theory was used in [19]-[20] to realize fuzzy representations of heuristic rules. In [19], all possible solutions were evaluated based on the membership values of the fuzzy objectives (loads restored) and the fuzzy constraints (line capacity limit, etc.). After calculating membership values for each solution, the best solution is found based on a maximin problem. The evaluation process in [19] was accelerated in [20] by using the heuristic procedure proposed in [12] and a fuzzy cause-effect network. Moreover, the fuzzy set theory can be used to model uncertainties of loads. In [21], loads of branch points were represented by fuzzy variables. According to mathematical operations on fuzzy variables, the heuristic algorithm proposed in [12] was used to find the solution with the minimum number of switching operations.

While heuristic algorithms can obtain a feasible solution quickly, they still require specialists' knowledge and it is hard to derive an optimal solution.

\section{3) Graph theory}

The distribution network can be interpreted to a graph, representing the buses as nodes and the lines connecting buses as edges of the graph. Therefore, the SR problem can be considered as a problem of finding spanning trees that represent possible radial structures of the network and satisfy operating constraints. Different techniques have been proposed in [22]-[26] to generate spanning trees. A cut set theory based spanning tree search algorithm was proposed in [22], in which a new tree can be generated by operating a pair of switches (a normally closed switch and a normally open switch) and the power flow calculation is conducted to evaluate all newly formed trees. In [23], according to the fundamental loop concept of the graph theory, a tree can be derived by removing one edge from every fundamental cycle. Likewise, all acquired trees are evaluated by power flow calculations. A similar tree search procedure based on the fundamental loop concept was proposed in [24] to form the structure of MGs. The author in [25] proposed an "interested tree" concept. An "interested tree" is a tree in which all loads are supplied by substations. After evaluating all "interested trees", the best full restoration plan can be reached. In [26], instead of substations, the nodes that are in energized areas and connect de-energized areas are considered as roots of trees, which means that the process of restoring faulted areas can be treated as a process of identifying multiple spanning trees.

Although the optimal solution can be obtained through an exhaustive search, the number of trees may be quite high for a large system, thus making the above algorithms unattractive.

\section{4) Meta-heuristic algorithm}

Many meta-heuristic algorithms have been developed to solve the formulated complex mixed integer programming (MIP) model, including genetic algorithm (GA) [27]-[28], tabe search (TS) [29], particle swarm optimization (PSO) 
algorithm [30], parallel-simulated annealing (PSA) algorithm [31] and so on. These algorithms have similar procedures but with different search strategies and encoding methods of solution variables.

In [31], four meta-heuristic algorithms (GA, TS, reactive TS, PSA) were described in detail and compared with respect to average execution time and the quality of solution obtained. In conventional meta-heuristic algorithms [31], a multiobjective problem is converted into an equivalent single objective problem by weighting factors, and the admissible switching sequence is not given. To overcome these disadvantages, some improved algorithms have been proposed. A non-dominated sorting genetic algorithm-II (NSGA-II) was adopted in [32] to solve the SR problem. The objectives are to minimize out of service areas, switching operations and network losses. The utilization of the nondominated sorting technique enables the algorithm to deal with multiple objectives without using weighting factors. A multi-objective revolutionary algorithm based methodology was proposed in [33], in which priority customers, priority switches and switching sequence are considered. In addition, the methodology uses the node-depth encoding method to represent solution variables, which always ensures the radial topology of the network. In [34], an interactive fuzzy satisfying method combining the fuzzy set theory and GA was proposed to deal with multi-objective nature of the problem. Firstly, values of multiple objectives are normalized by their corresponding membership functions, and the decision maker sets satisfied value $[0,1]$ for each objective. Then, the GA is used to find the solution whose membership values are close to the preset values. Finally, according to the satisfactory level of the solution found, the decision maker can reset their satisfied values and find a new solution.

Meta-heuristic algorithms do not require specialist' knowledge, however, they require extensive computational time when applied to a large system and cannot ensure an optimal solution.

\section{5) Mathematical programming}

Expert systems and heuristic algorithms were combined with mathematical programming in [35]-[36]. The author in [35] first used the generic knowledge of expert to divide a whole network into several sub-networks. Then, each subnetwork is formulated as a mixed integer linear programming (MILP) problem. In [36], the mathematical model was used when the simple heuristic search algorithm is unable to find a feasible solution. In this context, a "local network" is found and formulated as a MILP problem.

In [37]-[40], more sophisticated mathematical models for SR were developed. Two different formulations of the mixed integer non-linear programming (MINLP) problem for the unbalanced distribution network were developed in [37]. The first formulation uses the bus injection model and the second formulation uses the branch flow model. A non-linear solver LINGO is used to solve these two MINLP problems. It is difficult to solve the MINLP problem due to its non-convex and non-linear characteristics. The author in [38] relaxed the original MINLP problem into a mixed integer second order cone programming problem that can be solved efficiently and ensure the optimality of the solution. In [39], a two-stage strategy was proposed. The first stage uses piecewise linearization to transform the MINLP problem into a MILP problem that is solved in order to obtain the binary decision variables (topology of the network). In the second stage, a nonlinear programming problem is formulated based on the topology found to adjust the steady state operating point. A multi-time step MINLP problem considering dispathchable DG units was proposed in [40] to determine the optimal switching sequence. Similarly, the formulated MINLP problem is converted into a MILP problem though linearization techniques.

In addition, the dynamic programming (DP) technique has been proposed to determine the switching sequence. The author in [41] used the DP to determine the restoration sequence of feeders in outage areas for minimizing unserved energy during the duration of restoration. A two-phase strategy was proposed in [42], in which the first phase finds the target configuration through the GA algorithm, and the second phase determines the optimal sequence of switching operations using the DP.

Uncertainties of DG units and loads have also been studied. The author in [43] proposed a robust restoration model that has two-stage objective and has the form of maximizing a minimax problem. The formulated MILP problem is solved by a column-and-constrained generation method and an optimal decision (target configuration) that can restore as many loads as possible in the worst-case fluctuation scenario of outputs of DG units is derived. The author in [44] formulated an information gap decision theory based robust restoration decision-making model that takes into account uncertainties of DG units and loads. For a given bounded uncertain set, the solution found can guarantee that operating constraints are not violated and the amount of loads restored would not fall below a specified threshold. In [45], uncertainties of loads and pay back effect were modeled by fuzzy variables and a fuzzy mixed integer programming model was developed to perform risk management for SR.

Several mathematical models have been developed to solve the MG-aid SR problem. A comprehensive strategy with DG units and ESSs was proposed in [46], in which the normal operating condition and self-healing condition are considered. In the normal condition, a rolling-horizon MINLP model is developed to minimize operation costs and deal with uncertainties of DG units. However, the self-healing condition aims to optimally sectionalize the network into several MGs. A sequential restoration strategy was proposed in [47] to determine the switching sequence of constructing MGs. In the strategy, some typical components in the distribution network, such as voltage regulators, transformers and capacitor banks, are modelled. The author in [5] used the same sequential restoration strategy as the one in [47] while considering intertemporal constraints, like loads under cold load pick-up conditions. The bender decomposition technique was used in [48] to decompose the SR problem into a master problem and a slave problem. The master problem is to minimize load shedding in the reconfiguration work, and the slave problem is to minimize line losses in the islanding mode, namely the MGs mode. 
Although mathematical programming based methods can acquire an optimal solution under specified operating constraints, the solution time often exceeds practically allocated computation time for a large-scale problem due to the combinatorial nature.

\section{B. Decentralized method}

The above methods solve the SR problem from a centralized point of view. They requires a control center that has expensive computing capacity and a communication system that has low latency and can transfer a large amount of data. In addition, centralized methods suffer from a single point failure risk. To overcome these disadvantages, the multiagent system (MAS) is developed to distribute the intelligence and control actions to the component level [2].

In [49], a number of bus agents and a single facilitator agent were developed. The purpose of the bus agent is to restore the loads directly connected to its associated bus, and the facilitator agent aims to facilitate the negotiations between bus agents. However, the work does not consider the load priority and the presence of DGs. Moreover, the negotiation process mainly replies on only one agent (facilitator agent), which actually makes the MAS centralized. A fully decentralized MAS was proposed in [50], in which three types of agents, namely switch agents, load agents and generator agents, are developed. These agents have access to the local data and only communicate with their neighboring agents. In addition, the work takes into consideration of the load priority, thus full restoration and partial restoration can be reached. A two-layer MAS considering the presence of DG units was developed in [51]. In the first layer, zone agents monitor the fault, make simple calculations and implement control actions. In the second layer, feeder agents are assigned to negotiate. In the work, decision-making for all faults on the same feeder mainly replies on the sole feeder agent located at the feeder, which suffers from low reliability. To overcome such a disadvantage, the author in [52] proposed four types of zone agents including fault zone agents, down zone agents, zone tie agents and healthy zone agents. After a fault occurs, each zone gent identifies itself as one of the above four types of agents according to its location with respect to the fault. The fault zone agent is the decision-making agent. Therefore, different decision-making agents will be activated according to different faults. In [53], load agents, feeder agents and regulator agents were developed. Among them, the regulator agent aims to regulate overall nodal voltages of the network by moving the tap of OLTC. In addition, the fuzzy set theory is used in the decision-making process to determine the optimal supporting feeder. In addition, the fuzzy set theory is used in the decision-making process to determine the optimal supporting feeder.

The MG based MASs have also been developed. An agent based restoration strategy with the supports of ESSs was developed in [54], in which the normal condition and abnormal condition are considered. In the normal condition, ESSs are used to improve the voltage profile and reduce the line loss. In the abnormal operation, ESSs are considered as generation resources, and switch agents and energy storage agents are coordinated to form MGs to restore loads. A MAS restoration strategy considering the $\mathrm{V} 2 \mathrm{G}$ feature of $\mathrm{EV}$ was proposed in [6], in which four kinds of agents, namely load agents, aggregator agents, DG agents and switch agents, are developed. After receiving restoration requests from load agents, the aggregator agents will use available $\mathrm{V} 2 \mathrm{G}$ power to restore loads locally. Then, DG units in the islanding mode will supply the remaining unrestored loads.

The MAS based method can overcome the disadvantages of centralized methods by distributing control actions to the component level. However, the agent based decision-making process is based on some simple heuristic rules, which cannot ensure the optimality of the solution.

\section{CONCLUSION}

This paper reviews the existing methods for SR in distribution networks. These methods have two ways of implementation, namely centralized ways and decentralized ways, and use one or more techniques to obtain the SR plan. The used techniques are expert systems, heuristic algorithms, meta-heuristic algorithms, graph theory, multi-agent systems and mathematical programming. In addition, the advantages and disadvantages of these techniques are also given.

\section{REFERENCE}

[1] A. Coelho, A. B. Rodrigues, and M. G. Da Silva, "Distribution network reconfiguration with reliability constraints," in Proc. Int. Conf. Power Syst. Technol., vol. 2. Singapore, Nov. 2004, pp. 1600-1606.

[2] A. Zidan et al., "Fault detection, isolation, and service restoration in distribution systems: state-of-the-art and future trends," IEEE Trans. on Smart Grid, vol. 8, no. 5, pp. 2170-2185, Sept. 2017.

[3] N. A. Latare, S. S. Bhat and I. Srivastava, "Literature review of service restoration in distribution system," 2017 2th Int. Conf. on Elect. Comput. and Commun. Technol. (ICECCT), Coimbatore, 2017, pp. 1-6.

[4] B. Chen, C. Chen, J. Wang and K. L. Butler-Purry, "Multi-time step service restoration for advanced distribution systems and microgrids," IEEE Trans. on Smart Grid, to be published.

[5] A. Sharma, D. Srinivasan and A. Trivedi, "A decentralized multiagent system approach for service restoration using DG islanding," IEEE Trans. on Smart Grid, vol. 6, no. 6, pp. 2784-2793, Nov. 2015.

[6] D. S. Sanches, J. B. A. London, Jr., and A. C. B. Delbem, "Multiobjective evolutionary algorithm for single and multiple fault service restoration in large-scale distribution systems," Elect. Power Syst. Res., vol. 110, pp. 144-153, May 2014.

[7] S. Srivastava and K. L. Butler-Burry, "Expert-system method for automatic reconfiguration for restoration of shipboard power systems," in IEE Proc. Gene. Transm. Distrib., vol. 153, no. 3, pp. 253-260, 11 May 2006.

[8] C. C. Liu, S. J. Lee and S. S. Venkata, "An expert system operational aid for restoration and loss reduction of distribution systems," IEEE Trans. on Power Syst., vol. 3, no. 2, pp. 619-626, May 1988.

[9] S. J. Lee, K. H. Kim, H. Y. Kim, J. K. Lee, and K. Y. Nam, "Expert system-aided service restoration in distribution automation", in Proc. IEEE Int. Conf. Syst. Man Cybern., vol. 1. Chicago, IL, USA, Oct. 1992, pp. 157-161.

[10] Chao-Shun Chen, Chia-Hung Lin and Hung-Ying Tsai, "A rule-based expert system with colored petri net models for distribution system service restoration," IEEE Trans. on Power Syst., vol. 17, no. 4, pp. 1073-1080, Nov. 2002.

[11] M. S. Tsai, "Development of an object-oriented service restoration expert system with load variations," IEEE Trans. on Power Syst., vol. 23, no. 1, pp. 219-225, Feb. 2008.

[12] Y. Y. Hsu et al., "Distribution system service restoration using a heuristic search approach," IEEE Trans. on Power Del., vol. 7, no. 2, pp. 734-740, Apr. 1992.

[13] Karen Nan Miu, Hsiao-Dong Chiang and R. J. McNulty, "Multi-tier service restoration through network reconfiguration and capacitor 
control for large-scale radial distribution networks," IEEE Trans. on Power Syst., vol. 15, no. 3, pp. 1001-1007, Aug. 2000.

[14] M. R. Kleinberg, K. Miu and H. D. Chiang, "Improving Service Restoration of Power Distribution Systems Through Load Curtailment of In-Service Customers," IEEE Trans. on Power Syst., vol. 26, no. 3, pp. 1110-1117, Aug. 2011.

[15] D. Shirmohammadi, "Service restoration in distribution networks via network reconfiguration," IEEE Trans. on Power Del., vol. 7, no. 2, pp. 952-958, Apr. 1992.

[16] A. L. Morelato and A. J. Monticelli, "Heuristic search approach to distribution system restoration," IEEE Trans. on Power Del., vol. 4, no. 4, pp. 2235-2241, Oct. 1989.

[17] J. S. Wu, K. L. Tomsovic and C. S. Chen, "A heuristic search approach to feeder switching operations for overload, faults, unbalanced flow and maintenance," IEEE Trans. on Power Del, vol. 6, no. 4, pp. 1579-1586, Oct. 1991.

[18] A. Botea, J. Rintanen and D. Banerjee, "Optimal reconfiguration for supply restoration with informed A ${ }^{\text {ast }}$ search," IEEE Trans. on Smart Grid, vol. 3, no. 2, pp. 583-593, Jun. 2012.

[19] Yuan-Yih Hsu and Han-Ching Kuo, "A heuristic based fuzzy reasoning approach for distribution system service restoration," IEEE Trans. on Power Del., vol. 9, no. 2, pp. 948-953, Apr. 1994.

[20] Chao-Ming Huang, "Multiobjective service restoration of distribution systems using fuzzy cause-effect networks," IEEE Trans. on Power Syst., vol. 18, no. 2, pp. 867-874, May 2003.

[21] Han-Ching Kuo and Yuan-Yih Hsu, "Distribution system load estimation and service restoration using a fuzzy set approach," IEEE Trans. on Power Del., vol. 8, no. 4, pp. 1950-1957, Oct. 1993.

[22] J. Li, X. Y. Ma, C. C. Liu and K. P. Schneider, "Distribution system restoration with microgrids using spanning tree search," IEEE Trans. on Power Syst., vol. 29, no. 6, pp. 3021-3029, Nov. 2014.

[23] E. Drayer, N. Kechagia, J. Hegemann, M. Braun, M. Gabel and R. Caire, "Distributed self-healing for distribution grids with evolving search space," IEEE Trans. on Power Del., to be published.

[24] M. Zadsar, M. R. Haghifam and S. M. Miri Larimi, "Approach for selfhealing resilient operation of active distribution network with microgrid," IET Gener., Transm. Distrib., vol. 11, no. 18, pp. 46334643, 2017.

[25] N. D. R. Sarma, V. C. Prasad, K. S. Prakasa Rao and V. Sankar, "A new network reconfiguration technique for service restoration in distribution networks," IEEE Trans. on Power Del., vol. 9, no. 4, pp. 1936-1942, Oct. 1994

[26] S. Dimitrijevic and N. Rajakovic, "Service restoration of distribution networks considering switching operation costs and actual status of the switching equipment," IEEE Trans. on Smart Grid, vol. 6, no. 3, pp. 1227-1232, May 2015.

[27] A. Augugliaro, L. Dusonchet, and E. R. Sanseverino, "Service restoration in compensated distribution networks using a hybrid genetic algorithm," Elect. Power Syst. Res., vol. 46, no. 1, pp. 59-66, 1998.

[28] W. P. Luan, M. R. Irving, and J. S. Daniel, "Genetic algorithm for supply restoration and optimal load shedding in power system distribution networks," in Proc. Inst. Elect. Eng. Gener. Transm. Distrib., vol. 149, no. 2, pp. 145-151, Mar. 2002.

[29] S. Toune, H. Fudo, T. Genji, Y. Fukuyama and Y. Nakanishi, "A reactive tabu search for service restoration in electric power distribution systems," 1998 IEEE Int. Conf. on Evolutionary Comput. Proc., Anchorage, AK, 1998, pp. 763-768.

[30] D. Chen, X. Chen, J. Liu, X. Dong and Y. Liao, "Service restoration study of distribution system with distributed generators based on particle swarm optimization,” 2011 Int. Conf. on Advanced Power Syst. Automation and Protection, Beijing, 2011, pp. 1176-1181.

[31] S. Toune, H. Fudo, T. Genji, Y. Fukuyama and Y. Nakanishi, "Comparative study of modern heuristic algorithms to service restoration in distribution systems," IEEE Trans. on Power Del., vol. 17, no. 1, pp. 173-181, Jan. 2002.

[32] Y. Kumar, B. Das and J. Sharma, "Multiobjective, multiconstraint service restoration of electric power distribution system with priority customers," IEEE Trans. on Power Del., vol. 23, no. 1, pp. 261-270, Jan. 2008.

[33] L. T. Marques, A. C. B. Delbem and J. B. London., "Service Restoration with Prioritization of Customers and Switches and
Determination of Switching Sequence," IEEE Trans. on Smart Grid, to be published.

[34] Y. T. Hsiao and C. Y. Chien, "Enhancement of restoration service in distribution systems using a combination fuzzy-GA method," IEEE Trans. on Power Syst., vol. 15, no. 4, pp. 1394-1400, Nov. 2000.

[35] T. Nagata, H. Sasaki and R. Yokoyama, "Power system restoration by joint usage of expert system and mathematical programming approach," IEEE Trans. on Power Syst., vol. 10, no. 3, pp. 1473-1479, Aug. 1995.

[36] R. M. Ciric and D. S. Popovic, "Multi-objective distribution network restoration using heuristic approach and mix integer programming method," Elect. Power Energy Syst., vol. 22, pp. 497-505, 2000.

[37] S. Khushalani, J. M. Solanki and N. N. Schulz, "Optimized restoration of unbalanced distribution systems," IEEE Trans. on Power Syst., vol. 22, no. 2, pp. 624-630, May 2007.

[38] R. Romero, J. F. Franco, F. B. Leão, M. J. Rider and E. S. de Souza, “A new mathematical model for the restoration problem in balanced radial distribution systems," IEEE Trans. on Power Syst., vol. 31, no. 2, pp. 1259-1268, Mar. 2016.

[39] P. L. Cavalcante et al., "Centralized self-healing scheme for electrical distribution systems," IEEE Trans. on Smart Grid, vol. 7, no. 1, pp. 145-155, Jan. 2016

[40] J. C. Lopez, J. F. Franco, M. J. Rider and R. Romero, "Optimal restoration/maintenance switching sequence of unbalanced three-phase distribution systems," IEEE Trans. on Smart Grid, to be published.

[41] R. Perez-Guerrero, G. T. Heydt, N. J. Jack, B. K. Keel and A. R. Castelhano, "Optimal restoration of distribution systems using dynamic programming," IEEE Trans. on Power Del, vol. 23, no. 3, pp. 15891596, Jul. 2008.

[42] P.M. S. Carvalho, L. A. F. M. Ferreira, and L. M. F. Barruncho, "Optimization approach to dynamic restoration of distribution systems," Elect. Power Energy Syst., vol. 29, pp. 222-229, 2007.

[43] X. Chen, W. Wu and B. Zhang, "Robust restoration method for active distribution networks," IEEE Trans. on Power Syst., vol. 31, no. 5, pp. 4005-4015, Sept. 2016.

[44] K. Chen, W. Wu, B. Zhang and H. Sun, "Robust restoration decisionmaking model for distribution networks based on information gap decision theory," IEEE Trans. on Smart Grid, vol. 6, no. 2, pp. 587597, Mar. 2015

[45] D. S. Popovic and Z. N. Popovic, "A risk management procedure for supply restoration in distribution networks," IEEE Trans. on Power Syst., vol. 19, no. 1, pp. 221-228, Feb. 2004.

[46] Z. Wang and J. Wang, "Self-healing resilient distribution systems based on sectionalization into microgrids," IEEE Trans. on Power Syst., vol. 30, no. 6, pp. 3139-3149, Nov. 2015.

[47] B. Chen, C. Chen, J. Wang and K. L. Butler-Purry, "Sequential service restoration for unbalanced distribution systems and microgrids," IEEE Trans. on Power Syst., vol. 33, no. 2, pp. 1507-1520, Mar. 2018.

[48] Junhong Guo and Longjun Wang, "Self-healing resilience strategy to active distribution network based on benders decomposition approach," 2016 IEEE PES Asia-Pacific Power and Energy Eng. Conf. (APPEEC), Xi'an, 2016, pp. 1491-1495.

[49] T. Nagata and H. Sasaki, "A multi-agent approach to power system restoration," IEEE Trans. on Power Syst., vol. 17, no. 2, pp. 457-462, May 2002.

[50] J. M. Solanki, S. Khushalani and N. N. Schulz, "A multi-agent solution to distribution systems restoration," IEEE Trans. on Power Syst., vol. 22, no. 3, pp. 1026-1034, Aug. 2007.

[51] A. Zidan and E. F. El-Saadany, "A cooperative multiagent framework for self-healing mechanisms in distribution systems," IEEE Trans. on Smart Grid, vol. 3, no. 3, pp. 1525-1539, Sept. 2012.

[52] A. Abel Hafez, W. A. Omran and Y. G. Higazi, "A decentralized technique for autonomous service restoration in active radial distribution networks," IEEE Trans. on Smart Grid, to be published.

[53] A. Elmitwally, M. Elsaid, M. Elgamal and Z. Chen, "A fuzzymultiagent service restoration scheme for distribution system with distributed generation," EEE Trans. on Sustain. Energy, vol. 6, no. 3, pp. 810-821, Jul. 2015.

[54] C. P. Nguyen and A. J. Flueck, "Agent based restoration with distributed energy storage support in smart grids," IEEE Trans. on Smart Grid, vol. 3, no. 2, pp. 1029-1038, Jun. 2012. 